\title{
A gamma beam profile imager for ELI-NP Gamma Beam System
}

\author{
P. Cardarelli ${ }^{\mathrm{b}}$, G. Paternò ${ }^{\mathrm{b}, *}$, G. Di Domenico ${ }^{\mathrm{a}, \mathrm{b}}$, E. Consoli $^{\mathrm{a}, \mathrm{b}}$, M. Marziani $^{\mathrm{a}, \mathrm{b}}$, M. Andreotti $^{\mathrm{b}}$, \\ F. Evangelisti ${ }^{\text {b }}$, S. Squerzanti ${ }^{\text {b }}$, M. Gambaccini ${ }^{\text {a,b }}{ }^{\text {, S. Albergo }}{ }^{\text {c,e }}$, G. Cappello $^{c, e}$, A. Tricomi ${ }^{\text {c,e }}$, \\ M. Veltri ${ }^{\text {f,d }}$, O. Adriani ${ }^{\text {g,d }}$, R. Borgheresi ${ }^{g, d}$, G. Graziani ${ }^{\mathrm{d}}$, G. Passaleva ${ }^{\mathrm{d}}$, A. Serban ${ }^{\mathrm{d}, 1}$, \\ O. Starodubtsev ${ }^{\text {d }}$, A. Variola ${ }^{\text {h }}$, L. Palumbo ${ }^{i}$ \\ a Dipartimento di Fisica e Scienze della Terra, Università di Ferrara, Via G. Saragat 1, 44122 Ferrara, Italy \\ ${ }^{\mathrm{b}}$ INFN - Sez. Ferrara, Via G. Saragat 1, 44122 Ferrara, Italy \\ ' INFN - Sez. Catania, Via S. Sofia 64, 95123 Catania, Italy \\ d INFN - Sez. Firenze, Via G. Sansone 1, 50019 Sesto Fiorentino (FI), Italy \\ e Università di Catania, Via S. Sofia 64, 95123 Catania, Italy \\ ${ }^{\mathrm{f}}$ Università di Urbino, Via A. Saffi 2, 61029 Urbino (PU), Italy \\ 8 Università di Firenze, Via G. Sansone 1, 50019 Sesto Fiorentino (FI), Italy \\ ${ }^{h}$ INFN - Lab. Naz. di Frascati, Via E. Fermi 40, 00044 Frascati (RM), Italy \\ i Università "La Sapienza” di Roma, Piazzale A. Moro 5, 00185 Roma, Italy
}

\section{A R T I C L E I N F O}

\section{Keywords:}

Inverse Compton

Monochromatic radiation

$\mathrm{X}$ and gamma sources

Gamma beam diagnostics

Imager

\begin{abstract}
A B S T R A C T
The Gamma Beam System of ELI-Nuclear Physics is a high brilliance monochromatic gamma source based on the inverse Compton interaction between an intense high power laser and a bright electron beam with tunable energy. The source, currently being assembled in Magurele (Romania), is designed to provide a beam with tunable average energy ranging from 0.2 to $19.5 \mathrm{MeV}$, rms energy bandwidth down to $0.5 \%$ and flux of about $10^{8}$ photons/s. The system includes a set of detectors for the diagnostic and complete characterization of the gamma beam. To evaluate the spatial distribution of the beam a gamma beam profile imager is required. For this purpose, a detector based on a scintillator target coupled to a CCD camera was designed and a prototype was tested at INFN-Ferrara laboratories. A set of analytical calculations and Monte Carlo simulations were carried out to optimize the imager design and evaluate the performance expected with ELI-NP gamma beam. In this work the design of the imager is described in detail, as well as the simulation tools used and the results obtained. The simulation parameters were tuned and cross-checked with the experimental measurements carried out on the assembled prototype using the beam from an x-ray tube.
\end{abstract}

\section{Introduction}

ELI-Nuclear Physics (NP), currently being built in Magurele, Romania, is one of the three pillars of ELI (Extreme Light Infrastructures) European Project [1,2]. This facility will host the Gamma Beam System (GBS), an intense and monochromatic gamma source based on inverse Compton interaction between a high power laser and a high brightness electron beam produced by a warm LINAC. In 2014, EuroGammaS association, composed by many European research institutes and companies, leaded by INFN, won a tender to provide the design, manufacturing, installation and commissioning of ELI-NP-GBS [3-5]. The gamma beam is expected to feature energy ranging from 0.2 to $19.5 \mathrm{MeV}, 0.5 \% \mathrm{rms}$ bandwidth, flux of about $10^{8}$ collimated photons/s and unprecedented performance in terms of brilliance and spectral density. The GBS has a wide application prospect in many fields including nuclear physics, astrophysics, material science, and life sciences [6].

In order to cover the whole energy interval, the GBS will consist of two parallel beamlines, with two separated interaction points (IPs), one for gamma energies ranging from 0.2 to $3.5 \mathrm{MeV}$, and the other, after a further acceleration of the electron beam, will allow to reach energies from 3.5 to $19.5 \mathrm{MeV}$. The bandwidth requirement will be fulfilled by properly collimating the beam coming from the interaction points, exploiting the strong correlation between energy of the backscattered photons and scattering angle [7]. EuroGammaS collaboration will deliver, for each IP, a complete collimation and

\footnotetext{
* Corresponding author.

E-mail address: paterno@fe.infn.it (G. Paternò).

1 On leave from National Institute for Nuclear Physics and Engineering Horia Hulubei, Magurele, Romania.
} 
characterization system, which is currently being assembled and tested at Ferrara INFN section $[8,9]$. In order to cope with the unprecedented gamma beam specifications, innovative devices and techniques have been developed to measure and monitor the beam parameters useful for the to characterization of the source in terms of energy distribution, beam intensity, time structure and spatial profile $[10,11]$.

The gamma beam profile imager (GPI) has the task to acquire images of the spatial distribution of the collimated beam, allowing to verify its shape,size and uniformity. This information, in combination with the measure provided by the other detectors composing the characterization system will be used to check the alignment and operation of the collimation system.

In this paper we show the adopted design approach and precharacterization methodology together with the expected performance of the GPI.

\section{GPI design}

\subsection{Gamma beam features}

The gamma beam of the ELI-NP-GBS will be obtained from the Compton Back-Scattering interaction between pulses of a diode pumped $\mathrm{Yb}: Y A G$ laser and bunches of electrons, accelerated through a normal conducting linac consisting of $2 \mathrm{~S}$-band and $12 \mathrm{C}$-band RF structures subdivided in the two stages: High Energy (HE) and Low Energy (LE). The laser pulses will have a duration of 1.5 ps and a repetition rate of $100 \mathrm{~Hz}$. Due to a proper recirculating system, for each laser cycle, 32 bunches of electron will interact with the laser pulse at the same point [3]. As a result of these interactions, the gamma beam will have a time structure composed of 32 micro-pulses of about 1 ps with a separation of $16 \mathrm{~ns}$ representing a macro-pulse with a repetition rate of $100 \mathrm{~Hz}$ (see Fig. 1). The GPI aims to obtain an image of the average spatial distribution of the gamma beam in a time of the order of $1 \mathrm{~s}$. It will not be able to monitor the beam shot-to-shot.

As mentioned above, the required energy bandwidth will be obtained by collimating the gamma beam. The designed collimation system will produce beams with octagonal shape [8]. The GPI will be placed at a distance of $15.2 \mathrm{~m}$ (HE line) and $16.3 \mathrm{~m}$ (LE line) from the Interaction Point (IP), implying that the typical size of the beams will vary between about 1 to $11 \mathrm{~mm}$ (octagon's apothem), depending on the average energy and the energy bandwidth selected. In Fig. 2 the beam crosssection on the GPI obtained from simulations is shown in the case of $3 \mathrm{MeV}$ and $10 \mathrm{MeV}$ gamma beams. In both cases the collimation aperture was set to obtain an rms energy bandwidth of $0.5 \%$. The fluctuation in the beam intensity is purely statistical in nature, due to the relatively low number of inverse Compton interactions simulated. Indeed, the collimated beams are expected to feature a uniform intensity distribution.

\subsection{GPI layout}

The GPI must image gamma beams with variable size and brilliance, therefore it must allow to change its setting. Moreover, it has to fulfill the following requirements:

- provide images in a short time,

- spatial resolution down to $100 \mu \mathrm{m}$,

- usage of vacuum compatible materials,

- safe operation in a radiation environment.

The adopted solution is shown in Fig. 3 and described below. The GPI is composed of a cross vacuum chamber equipped with a mechanical actuator that allows to drive a target holder in a high vacuum $\left(10^{-7} \mathrm{mbar}\right)$ using a bellow. The screen holder supports interchangeable scintillator crystals (transparent media) that intercept the gamma beam at an angle of $45^{\circ}$. Outside of the vacuum, looking at the target through a quartz viewport, a CCD camera coupled with a proper photographic
Table 1

LYSO features.

\begin{tabular}{ll}
\hline Crystal structure & Monoclinic \\
\hline Density $\left(\mathrm{g} / \mathrm{cm}^{3}\right)$ & 7.2 \\
Radiation length $(\mathrm{cm})$ & 1.14 \\
Nuclear int. length $(\mathrm{cm})$ & 20.9 \\
Moliere radius $(\mathrm{cm})$ & 2.07 \\
Light yield $(\mathrm{ph} / \mathrm{MeV})$ & 25000 \\
Light peak emission $(\mathrm{nm})$ & 420 \\
Decay time (ns) & 40 \\
Refractive index (at $\left.\lambda_{\text {peak }}\right)$ & 1.82 \\
Radioactive & yes \\
Hygroscopic & no \\
Radiation hardness $(\mathrm{Gy})$ & $>10^{4}$
\end{tabular}

objective is installed. The viewport is oriented at $45^{\circ}$ with respect to the beam direction, allowing to acquire images of the target from a direction perpendicular to the target plan. The camera supporting frame includes a mirror, which reflects downwards the light coming out from the vacuum window. Also, the camera is mounted on a remotely controlled linear stage for fine focus adjustment. The entire system is enclosed in a dark box to avoid background signals from environmental light. Futhermore, the vacuum beamline is completely light-tight and the signal due to interaction laser leaks that through multiple reflections could reach the CCD is estimated to be negligible. The linear stage allows to move the position of the camera in a range of distances from the target that goes from $586 \mathrm{~mm}$ up to $800 \mathrm{~mm}$. This range of distances permits a safe operation of the CCD. Indeed, the expected dose rate in air at these locations was evaluated through a dedicated Geant 4 simulation tool including all the most relevant elements of the collimation and characterization line $[8,12]$ and resulted to be compatible with the dose rate allowed for radiation protection purposes and therefore suited for electronic devices.

\subsection{Scintillator selection}

The selection of the most convenient scintillator target is the result of a trade-off between conflicting requirements and has been carried out through a set of Monte Carlo simulations.

In order to have good images in a short time, the target used should have a good conversion efficiency and therefore should have a high density, high-Z, and feature a good light yield, which is the mean number of optical photons produced per unit of energy loss by a particle traveling through the scintillator. Moreover, the efficiency is strongly dependent on the target thickness, but the thickness cannot be increased arbitrarily without losing resolution.

Fig. 4 shows the light emission from various scintillator crystals of different thickness in the case of the $3 \mathrm{MeV}$ beam. Namely, a gamma beam with $0.5 \% \mathrm{rms}$ bandwidth, intensity of $3.6 \times 10^{8}$ photons $/ \mathrm{s}$ and divergence of $194 \mu \mathrm{rad}$. For each crystal, the total energy released by the gamma beam inside its volume was evaluated from the simulations. Then, the light emission was calculated by multiplying this value by light yield of the scintillator. This latter depends on the chemical composition, doping and crystalline quality of the material. For our estimation, we considered the mean value of light yield found in the literature for each crystal (see for instance [13-17]). Moreover, the fraction of light lost due to total internal reflection was taken into account.

Lutetium-yttrium oxyorthosilicate (LYSO) resulted by far the material producing more light and therefore it was chosen as the target of the GPI. In particular, we considered a Cerium-doped LYSO scintillator with chemical formula $\mathrm{Lu}_{1.9} \mathrm{Y}_{0.1} \mathrm{SiO}_{5}: \mathrm{Ce}(0.5 \%)$ produced by Epic-Crystal. Its characteristics were extracted from the datasheet provided by the manufacturer and various sources in the literature (see for instance [1821]). They are reported in Table 1.

Scintillators emit more light as their thickness increases, because the probability of energy deposition is higher. However, thicker samples 


\section{ARTICLE IN PRESS}
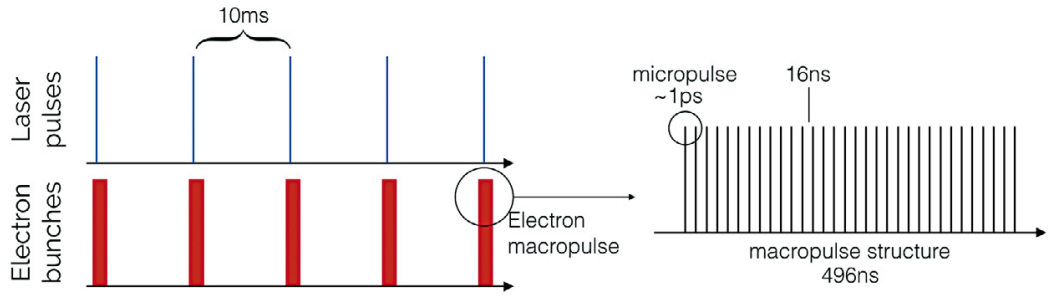

Fig. 1. Temporal structure of the colliding beams.
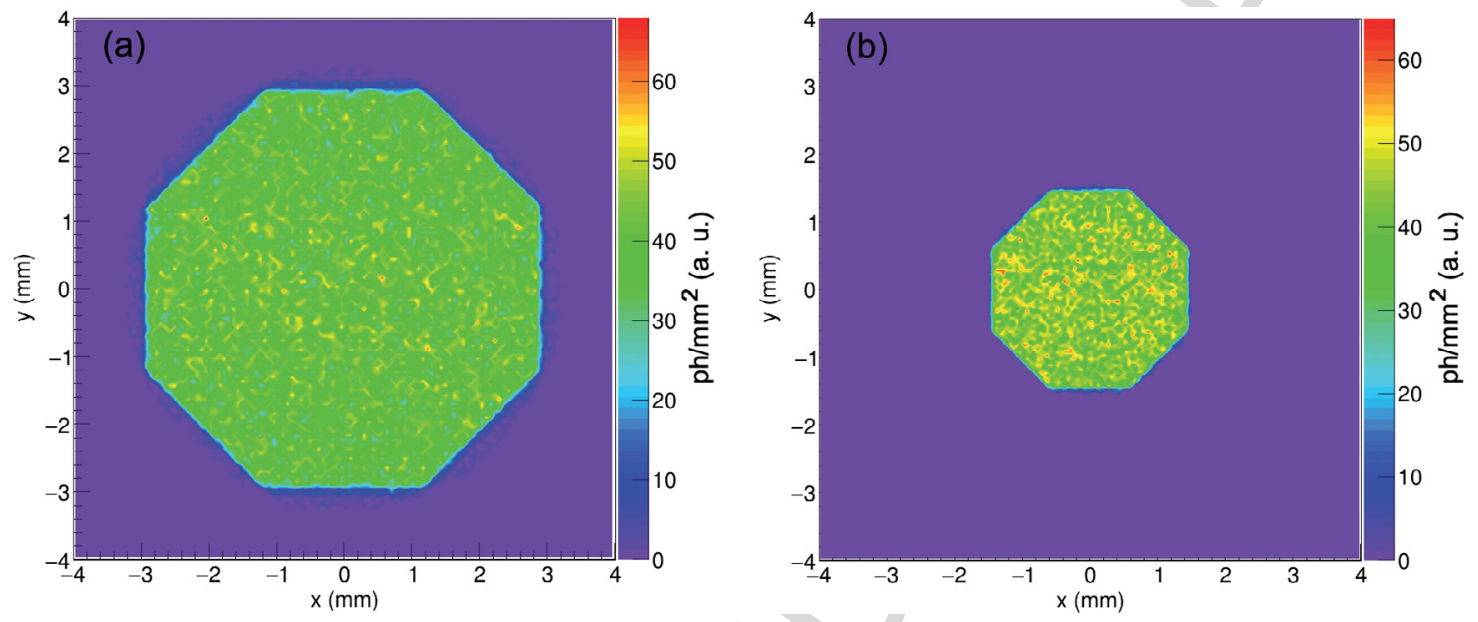

Fig. 2. Cross-section of the collimated gamma beam impinging on the GPI as it results from simulation. (a) $3 \mathrm{MeV}$ beam. (b) $10 \mathrm{MeV}$ beam.
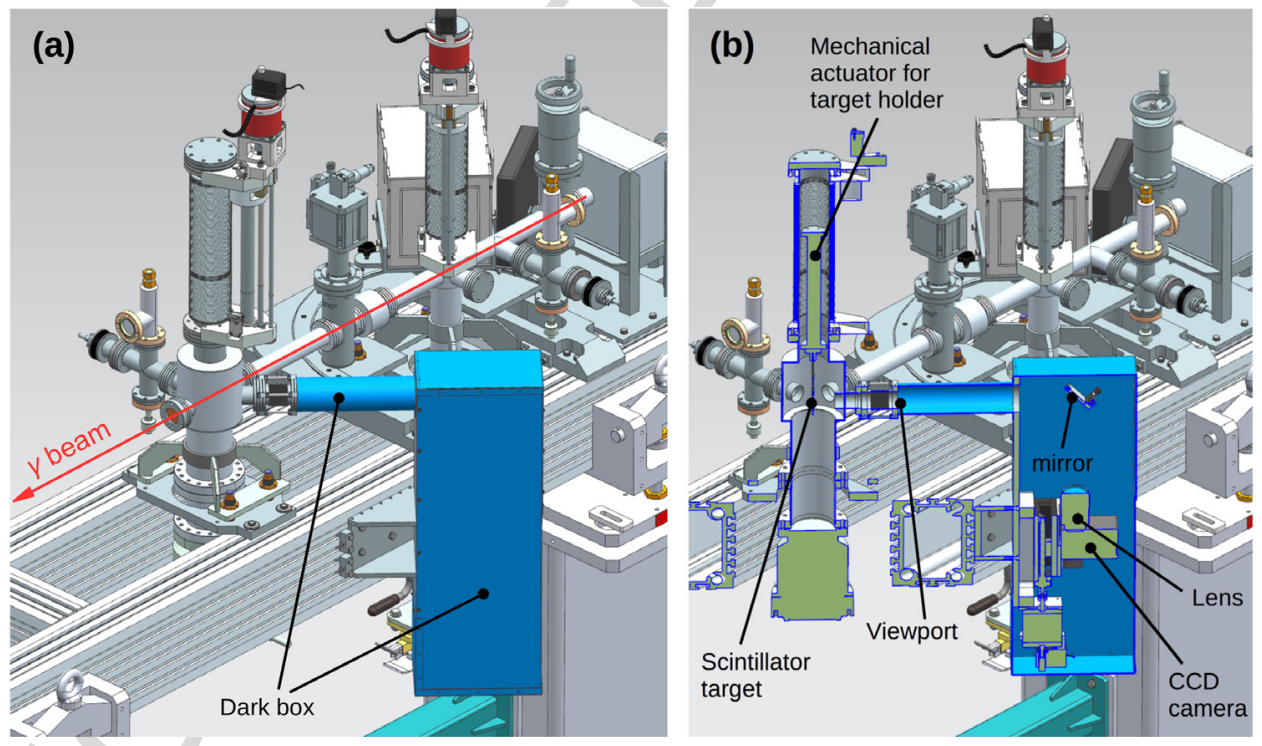

Fig. 3. Layout of the GPI. (a) Perspective view. (b) Cross-section.

lead to a degradation of the achievable image resolution. This is due to scattering and, moreover, to the fact that the region where the energy is deposited moves transversely as the gamma beam crosses the crystal at an angle of $45^{\circ}$. This effect is shown in Fig. 5, where the energy deposition distribution inside a LYSO crystal is reported for 2 different thicknesses and 2 different energies of the gamma beam. The resulting image on the CCD will be blurred along one axis. The blur rigorously depends on the energy deposition profile inside the scintillator, and therefore on the gamma beam energy, but it is of the order of magnitude of the crystal thickness. This effect combines with the resolution of the imaging system (lens + CCD) resolution, which depends on the acquisition parameters, such as lens aperture and pixel binning. For the GPI prototype described below, the intrinsic resolution was measured by irradiating frontally the LYSO crystal with X-rays from an X-ray tube and using a slit camera to obtain the Line Spread Function (LSF) and resulted to be between $80 \mu \mathrm{m}$ and $140 \mu \mathrm{m}$ (FWHM of the system LSF). Therefore, for scintillators thicker than few hundreds of microns, the blur due to the tilted irradiation is the resolution limiting factor. This target thickness provides a significant signal even at the lowest energy and a minimum resolution of $80 \mu \mathrm{m}$ along the vertical direction and $500 \mu \mathrm{m}$ along the longitudinal direction. In the case of beams with energy higher than 3.5 MeV, the beam cross-section gets considerably smaller, so a higher 


\section{ARTICLE IN PRESS}

resolution may be required also on the longitudinal axis. Considering that the specific energy deposition inside the scintillator increases, and therefore the signal expected on $\mathrm{CCD}$ gets higher, it may be preferable to use thinner scintillators to limit the blur effect and obtain images with a better resolution. For this reason, the GPI target holder will host a set of crystals with thickness between $100 \mu \mathrm{m}$ and $500 \mu \mathrm{m}$, allowing to enhance either the signal amplitude or the image resolution by selecting a suitable target.

The radiation hardness of LYSO, compared to the average dose released by ELI-NP-GBS working at the nominal conditions, allows a continuous irradiation for several days without a significant degradation of performance in terms of light yield and transparency. Therefore, considering the small fraction of time in which the target will be exposed to the beam during a routine use, it is possible to conclude that the degradation of performance due to radiation damage is not critical for several months of usage.

LYSO is slightly radioactive due to the $2.6 \%$ natural abundance of ${ }^{176} \mathrm{Lu}$ isotope, which has a half life of $\sim 2.2 \times 10^{10}$ years. However, considering the thickness and mass of the crystal used, the radioactivity is negligible and will not affect the operation of the detectors and the safety of handling.

(a)

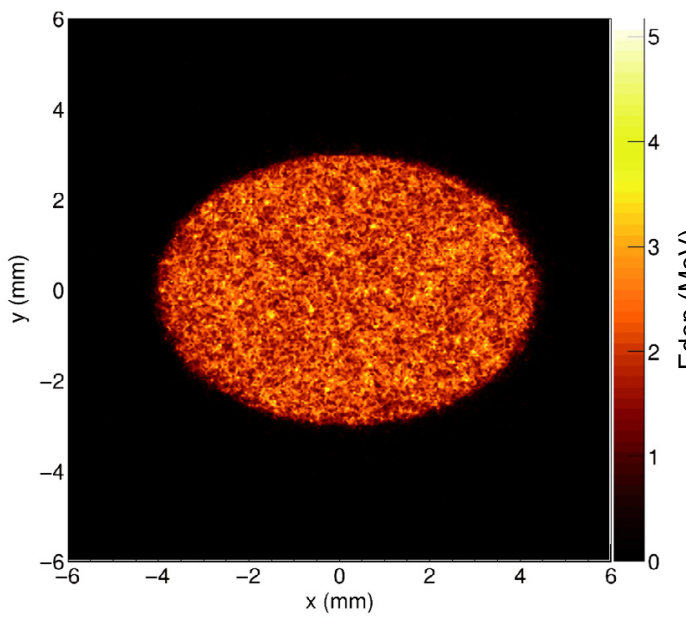

(c)

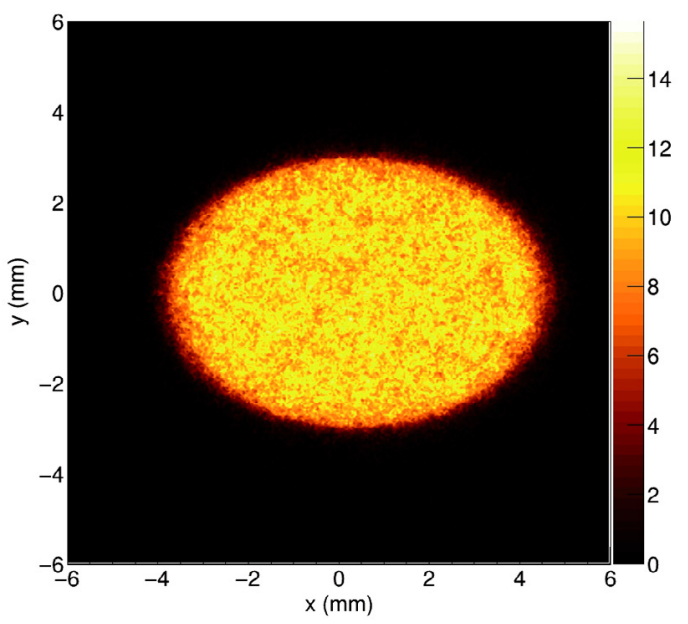

Nuclear Inst. and Methods in Physics Research, A $x x(x x x x) x x x-x x x$

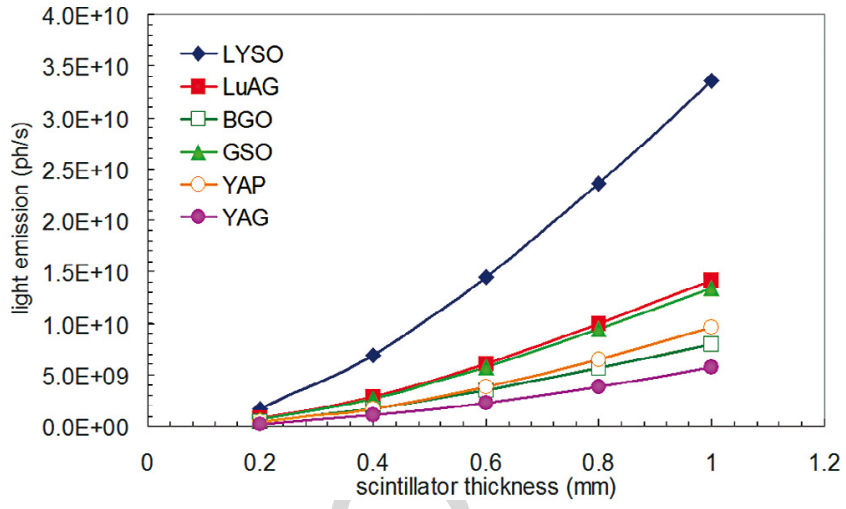

Fig. 4. Light emission from various crystals as a function of thickness for the $3 \mathrm{MeV}$ beam.

\section{Analytical model for performance estimation}

In order to select an imaging system providing the desired performance, a simple analytic model has been developed. The main goal of the model is to work out an expression for the signal expected on the

(b)

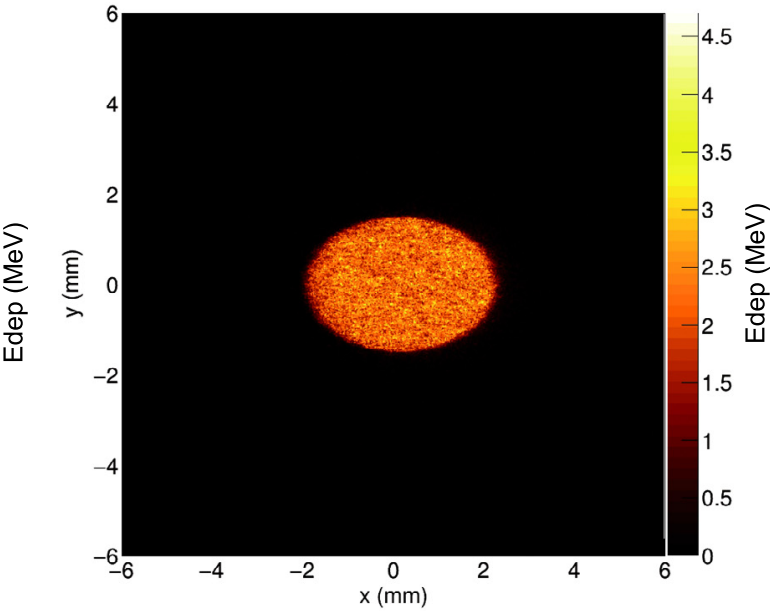

(d)

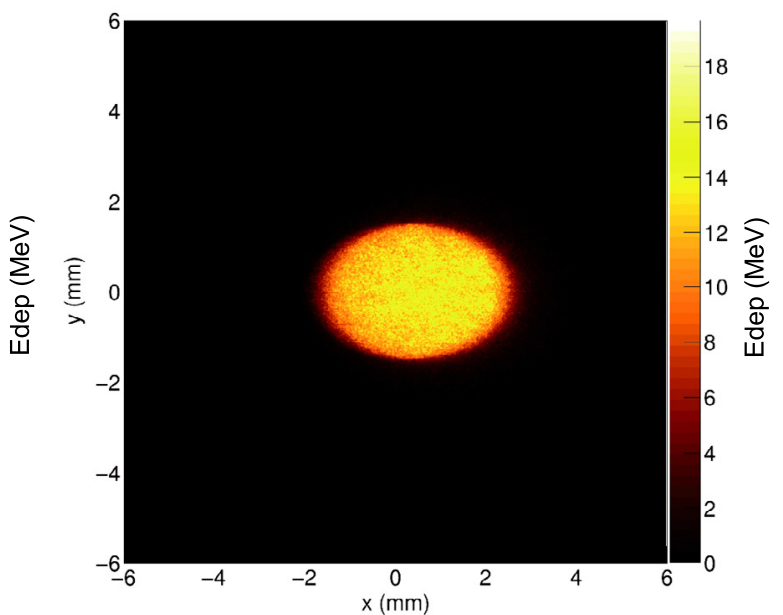

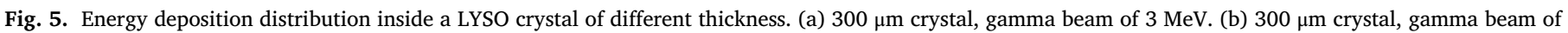

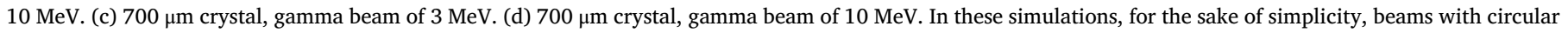
shape were considered. 


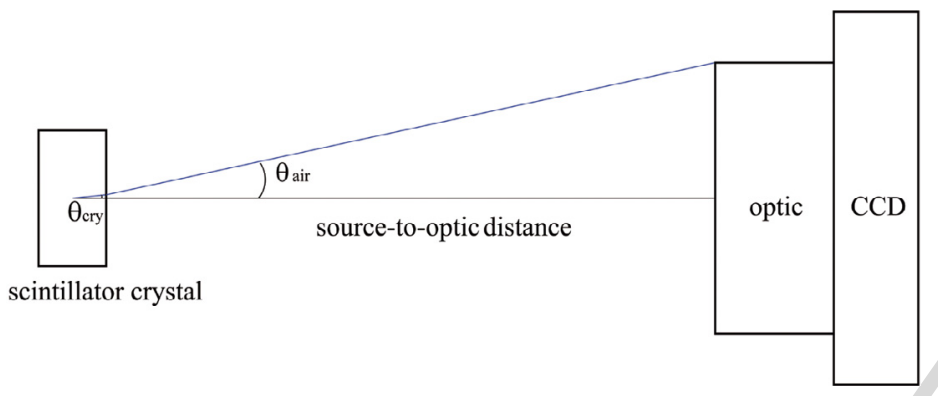

Fig. 6. Scheme used to evaluate the signal on the CCD.

CCD as a function of the system configuration. Fig. 6 shows a sketch of the system. A scintillator crystal with light yield $Y$, thickness $t$ and refractive index $n$ (at the emission peak wavelength) is irradiated by a gamma beam and emits optical photons (ph), which undergo refraction when they exit the crystal. Some of these photons are collected by an optic and focused on a CCD, which convert them in a gray level image.

The mean gray level per second of a pixel of the image can be written as

$\mathrm{GL}=\frac{E_{d e p} Y \varepsilon T c_{f} \Delta_{\mathrm{CCD}}^{2}}{A m^{2}}$,

where $E_{d e p}$ is the energy deposited in the unit of time by the gamma beam in a region of the scintillator of area $A, \varepsilon$ and $T$ are the collection efficiency and the transmission factor of the optic respectively, $c_{f}$ and $\Delta_{\mathrm{CCD}}$ are the gray level per incident photon $\mathrm{n}$ and the length of CCD pixel side respectively, and $m$ is the magnification ratio of the system, namely the ratio between image size and object size (in this case, the scintillation spot).

If we make the hypothesis that the scintillator-to-optic distance $p$ is much larger than the crystal thickness and the lens diaphragm diameter $D$, the small angle approximation can be used and the optic collection efficiency can be written as

$\varepsilon=\frac{\Omega}{4 \pi}=\frac{1-\cos \theta_{\text {cry }}}{2} \simeq \frac{\theta_{\text {cry }}^{2}}{4}=\frac{\theta_{\text {air }}^{2}}{4 n^{2}}=\frac{D^{2}}{16 n^{2} p^{2}}$.

Moreover, if we use the thin lens expression for the magnification ratio $m=\frac{f}{p-f}$,

and introduce the lens F-stop $F=f / D$, we end up with

$\varepsilon=\frac{1}{16 n^{2} F^{2}} \frac{m^{2}}{(1+m)^{2}}$,

which is widely used in the literature (See for instance [22]). The CCD $c_{f}$ coefficient can be written as

$c_{f}=Q E \cdot C T E \cdot G \cdot F F$,

where $Q E$ is the quantum efficiency (e/ph) at the scintillator peak emission wavelength, $C T E$ the charge transmission efficiency, $G$ the electronic Gain (GL/e), and $F F$ the fill factor, namely the ratio of active area and total area of the sensor. Using Eqs. (4) and (5), (1) becomes

$\mathrm{GL}=\frac{c_{f} \Delta_{\mathrm{CCD}}^{2}}{16} \frac{Y}{n^{2}} \frac{E_{d e p}}{A} \frac{T}{F^{2}} \frac{1}{(1+m)^{2}}$.

The energy deposited per second inside the scintillator by a gamma beam of energy $E$ can be evaluated via simulation or can be calculated through the following expression

$E_{d e p}=N E\left[\frac{\mu_{e n}(E) / \rho}{\mu(E) / \rho}\right](1-\exp (\mu(E) t))$,

where $\rho, \mu(E)$, and $\mu_{e n}(E)$ are the density, the linear attenuation coefficient and the absorption coefficient at energy $E$ of the scintillator, respectively, and $N$ is the number of photons per second of the beam.
From Eq. (6), it is possible to clearly identify the individual contribution of each system component to the signal on the CCD. As expected, the higher $c_{f}$ and $T$, namely the quality of the imaging system, the higher GL, moreover, GL increases if the lens aperture and the CCD pixel size are increased. The factor $Y / n^{2}$ accounts for the contribution of the scintillator while the factor $E_{d e p} / A$ depends both on the target and the gamma beam to be imaged. The higher the specific energy deposition inside the target, the higher GL. Therefore, the expected signal amplitude increases as the mean energy of the gamma beam increases. Finally, GL increases if the chosen magnification ratio decreases.

There are limitations to the range of useful magnification values. The lower bound is due to the required resolution of the imaging system in the object space $R_{\mathrm{obj}}$. In particular, by imposing that a detail of the image with size equal to $R_{\text {obj }}$ is imaged by at least 2 pixels of the camera, it follows that

$m \geq \frac{2 \Delta_{\mathrm{CCD}}}{R_{\mathrm{obj}}}$.

On the other hand, if we impose that the scintillator thickness is entirely inside the depth of field of the lens $D o F$

$D o F=2 \epsilon \frac{p^{2}}{D f} \geq t$

where $\epsilon$ is the diameter of the confusion circle, we obtain the upper bound for $m$

$m \leq \frac{b}{1-b} \quad b=\sqrt{\frac{2 \epsilon F}{t}}$.

If we set typical values for the system parameters, namely $t=0.5 \mathrm{~mm}$, $F=2, \varepsilon=5 \mu \mathrm{m}, \Delta_{\mathrm{CCD}}=5 \mu \mathrm{m}, R_{\mathrm{obj}} \approx 100 \mu \mathrm{m}$, we obtain $0.1 \leq m \leq 0.4$.

\section{Prototype test and model validation}

A simplified prototype of the designed GPI was assembled and tested in our laboratory using the photon beam from a Varian M-143T X-ray tube [23]. The experimental setup is shown in Fig. 7.

The used X-ray tube features a Beryllium window with a thickness of $0.63 \mathrm{~mm}$ and a nominal focal spot size of $0.1 \mathrm{~mm} \times 0.35 \mathrm{~mm}$. The source was powered by a $50 \mathrm{kHz}$, Metaltronica Compact MammoHF generator [24] with an adjustable voltage form 20 to $49 \mathrm{kV}$ and was operated in high current mode, which allows short exposition time, $5 \mathrm{~s}$ maximum, but current higher than $40 \mathrm{~mA}$. A $0.5 \mathrm{~mm}$ thick LYSO scintillator produced by Epic-Crystal was positioned in a dark box securing it with tape at a distance of about $200 \mathrm{~mm}$ from the X-ray tube. Collimators with hole of various diameters were used to limit the portion of target irradiated by the X-ray beam. The light emitted by the scintillator was focused through a Nikon Nikkor AF $85 \mathrm{~mm} / \mathrm{f1}$.4 D IF photographic lens [25] onto a Diffraction Limited SBIG STT-8300M CCD camera [26] whose specifications are listed in Table 2. The scintillator-to-lens distance was set to $667 \mathrm{~mm}$. The exact distance of the scintillator from the X-ray tube focus and the magnification of the system were measured by inserting an object of known size between the 


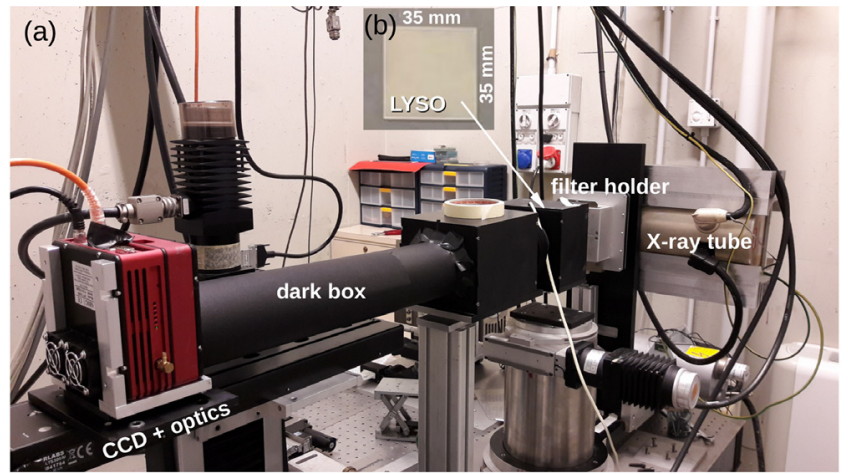

Fig. 7. (a) Picture of the experimental setup used to test the GPI prototype. (b) Picture of the scintillator crystal used.

Table 2

CCD main features.

\begin{tabular}{ll} 
Sensor size & $17.96 \mathrm{~mm} \times 13.52 \mathrm{~mm}$ \\
Pixel matrix & $3326 \times 2504$ \\
Pixel size & $5.4 \mu \mathrm{m} \times 5.4 \mu \mathrm{m}$ \\
Binning mode & $1 \times 1,2 \times 2,3 \times 3$ \\
Quantum eff. $(420 \mathrm{~nm})$ & $36 \%$ \\
Charge transfer eff. & 0.999995 \\
A/D Converter & $16 \mathrm{bit}$ \\
A/D Gain & $2.7 \mathrm{ADU} / \mathrm{e}^{-}$ \\
Full well capacity & $25000 \mathrm{e}^{-}$ \\
Read noise & $16 \mathrm{e}^{-}$ \\
Dark current & $1 \mathrm{e}^{-} / \mathrm{s} /$ pixel at $20{ }^{\circ} \mathrm{C}$ \\
\hline
\end{tabular}

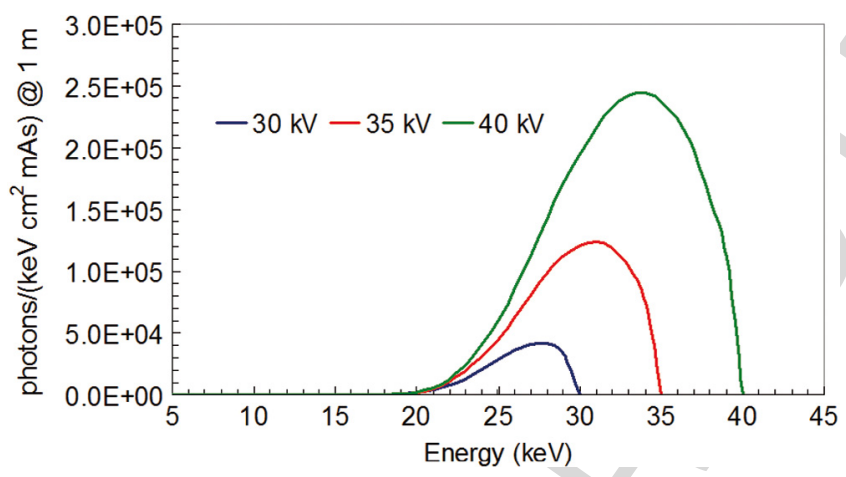

Fig. 8. Spectrum of the X-ray tube used to test the GPI prototype. The spectrum was calculated through the SpekCalc software [27] for various voltages. The spectrum is expressed as the photon energy fluence (photons $/\left(\mathrm{keV} \mathrm{cm}{ }^{2}\right)$ ) at $1 \mathrm{~m}$ from the X-ray tube focus for each $\mathrm{mAs}$ of the tube, which is the product of the anodic current $(\mathrm{mA})$ and the time of irradiation (s) and represents the electron charge $(\mathrm{mC})$ impinging on the anodic surface.

X-ray source and the scintillator and acquiring an image with the object positioned at 2 different distances from the scintillator. The distance of the scintillator from the X-ray tube focus resulted to be $232 \mathrm{~mm}$, while the magnification resulted to be 0.13 . During the measurements, the auto focus of the lens was not used, indeed, the image was focused by moving the whole optical system by a linear stage remotely controlled.

The X-ray source was set in such a way that, the signal obtained was of the same order of magnitude of that expected for the LE line of ELI-NP-GBS. In particular, we irradiated the scintillator for $1 \mathrm{~s} / \mathrm{shot}$ at 30,35 and $40 \mathrm{kV}$, using a filtration composed of a $5.1 \mathrm{~mm}$ thick $\mathrm{Al}$ foil plus a $0.1 \mathrm{~mm}$ thick $\mathrm{Cu}$ foil and increasing the anodic current from 10 to $30 \mathrm{~mA}$. The resulting X-ray spectrum is shown in Fig. 8.

In a first series of measures, we set the lens F-stop to $F=1.4$, the CCD to $2 \times 2$ binning mode and irradiated almost all the scintillator target (see Fig. 9).

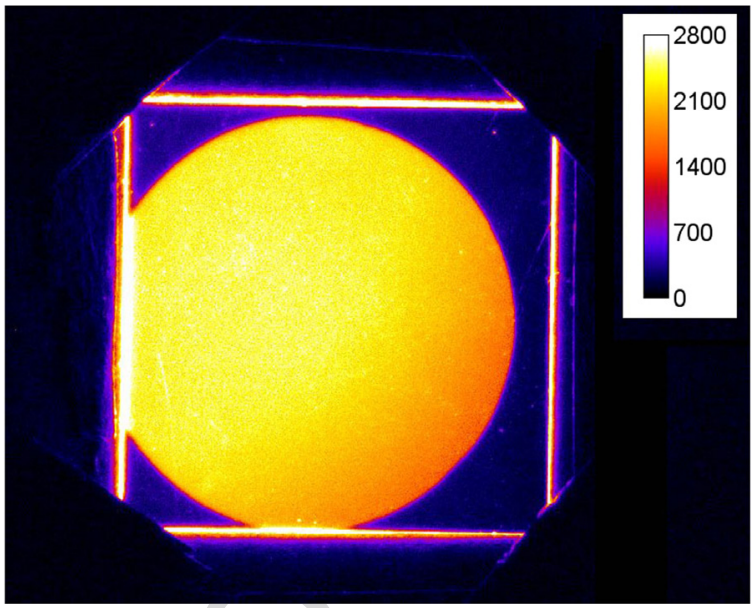

Fig. 9. False color image of the spot on CCD obtained with the X-ray tube set at $35 \mathrm{kV}$ and $30 \mathrm{~mA}$ and acquiring the signal for $1 \mathrm{~s}$. In this case the X-ray beam impinged normally on the scintillator surface passing through a collimator with a hole of $1.23 \mathrm{~cm}$ diameter. The light coming out from the edges of the crystal was due to the fact that they have a rounded profile and are not polished.

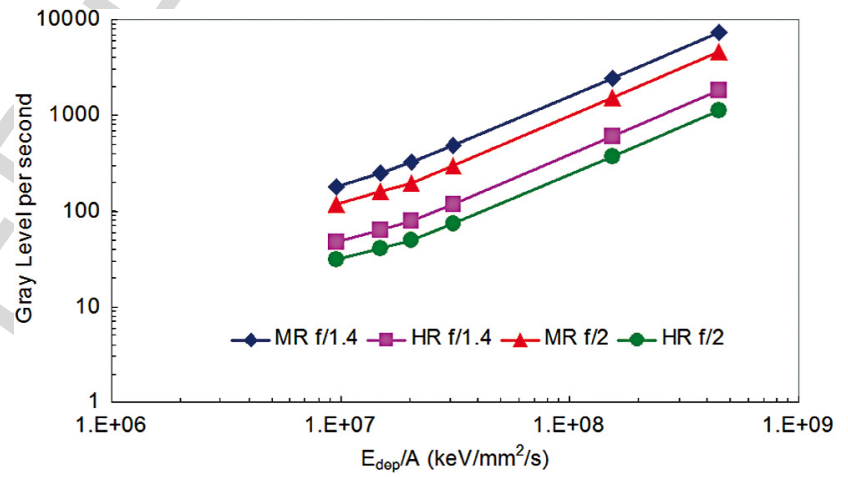

Fig. 10. Signal measured with various apertures of the lens diaphragm and binning configurations of the CCD.

The measured signal, namely the mean value of GL inside the spot on the CCD, was compared to the value estimated using Eq. (1) with $E_{d e p} / A$ calculated through both Eq. (7) and simulation. The obtained results are summarized in Table 3.

The optic transmission coefficient $T$ and the CCD fill factor $F F$, namely the parameters which were not exactly known a priori, were reasonably set to 0.8 and 0.95 , respectively. Indeed, using these values, the result provided by simulation and measure, for the case in which the X-ray tube was set to $40 \mathrm{kV}$ and $30 \mathrm{~mA}$, are in good agreement. This setting was chosen because the specific energy deposition inside the scintillator is approximately the same of that of the $3 \mathrm{MeV}$ beam of ELI-NP-GBS. From Table 3, where the average GL over the spot area on the CCD is reported, it is possible to note a good agreement between measurements and calculations for all settings and that simulations provide a better estimation than analytical calculations.

A further test was carried out by acquiring images with different apertures of the lens diaphragm and binning configurations of the CCD. In particular, two different F-stop values, 1.4 and 2 respectively, and two different binning modes $1 \times 1$ (HR) and $2 \times 2(\mathrm{MR})$ respectively, were considered. The results of the measurements are reported in Fig. 10 as a function of the specific energy deposition.

It is possible to note that, the signal scales almost linearly with the specific deposited energy and that it becomes about 4 times higher after we switch from HR to MR, as expected. A slight discrepancy was found 


\section{ARTICLE IN PRESS}

Table 3

CCD signal comparison.

\begin{tabular}{llccl}
\hline Voltage $(\mathrm{kV})$ & Nominal current $(\mathrm{mA})$ & Analytical $(\mathrm{GL})$ & Simulation $(\mathrm{GL})$ & Measure $(\mathrm{GL})$ \\
\hline 30 & 10 & 140 & 153 & $180 \pm 1$ \\
30 & 15 & 219 & 239 & $250 \pm 1$ \\
30 & 20 & 297 & 325 & $320 \pm 1$ \\
30 & 30 & 454 & 497 & $477 \pm 1$ \\
35 & 30 & 2264 & 2455 & $2454 \pm 7$ \\
40 & 30 & 6454 & 7174 & $7371 \pm 22$ \\
\hline
\end{tabular}

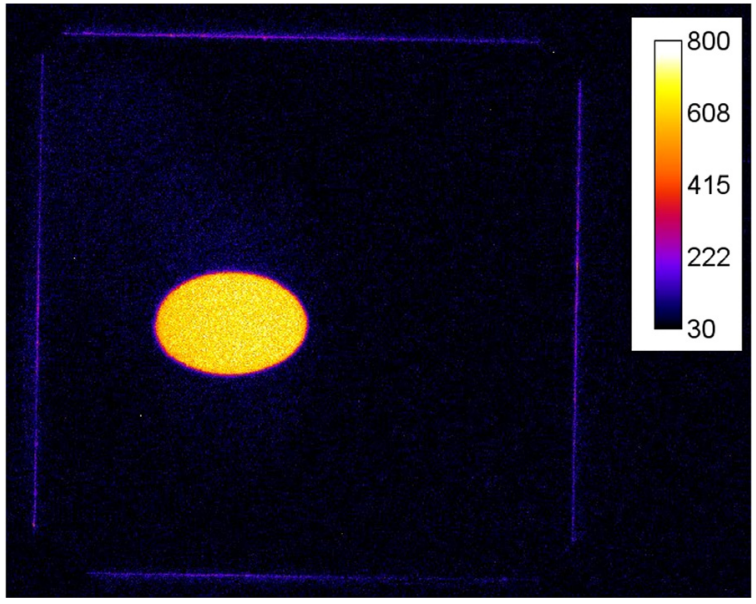

Fig. 11. False color image of the spot on CCD obtained with the X-ray tube set at $35 \mathrm{kV}$ and $30 \mathrm{~mA}$ and acquiring the signal for $1 \mathrm{~s}$. In this case the X-ray beam impinged at $45^{\circ}$ on the scintillator surface passing through a collimator with a hole of $3 \mathrm{~mm}$ diameter.

Table 4

Signal with ELI-NP-GBS beams.

\begin{tabular}{lc}
\hline$E_{\text {beam }}(\mathrm{MeV})$ & Signal $(\mathrm{GL})$ in $1 \mathrm{~s}$ \\
\hline 0.2 & 305 \\
3 & 2165 \\
10 & 24321 \\
19.5 & 51400 \\
\hline
\end{tabular}

when the lens F-stop if reduced from 2 to 1.4. Indeed, the signal does not double as expected, it gains only a factor 1.6. This discrepancy could be due to a not perfect tuning of the steps of the diaphragm aperture. However, it is possible to take into account this effect in our model introducing a simple correction factor.

A final test was carried out on the GPI prototype, to get closer to the real conditions of use. In this case, the X-ray tube was rotated by $45^{\circ}$ around the vertical axis and a collimator with a smaller hole was used. The acquired image is shown in Fig. 11 and features the expected elliptical shape.

\section{Monte Carlo simulation of ELI-NP-GBS beam}

Once the proposed model was validated, the expected signal with the ELI-NP-GBS beam was calculated through simulations. First, the spatial distribution of energy deposition inside a $0.5 \mathrm{~mm}$ thick LYSO crystal by collimated gamma beams of various energy was calculated through a set of simulations using the aforementioned dedicated Geant4 tool. Then, the signal on the CCD was calculated using the analytical model described in the previous section. Table 4 reports the results obtained for various gamma beam energies, setting $p=667 \mathrm{~mm}, F=1.4$ and $2 \times 2$ binning mode.

The signal results to be far above the expected readout and thermal noise of about 45 GL for the overall range of energy. Moreover, it can be seen that, the lens F-stop and CCD binning configuration can be changed

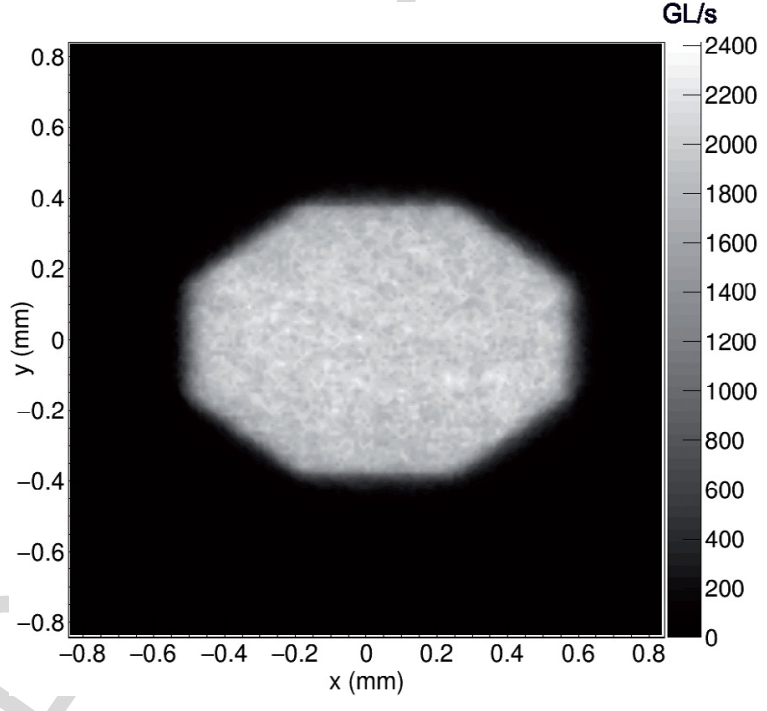

Fig. 12. Simulated image of the $3 \mathrm{MeV}$ beam on the CCD.

to 2 and $1 \times 1$ respectively, for the higher energy beams, due to their higher specific energy deposition in LYSO. In this way, the achievable spatial resolution can be increased.

The expected image on the CCD was also simulated. A dedicated paraxial ray-tracing code was developed in matlab language [28]. Starting from the energy deposition distribution calculated before, a number of optical photons were generated randomly inside the scintillator and tracked to the optic and the CCD using the matrix approach. Since the detailed configuration of the real lens is not known, an equivalent lens, namely a thin lens with the same aperture diameter and providing the same magnification of the real lens, was considered.

Fig. 12 reports the simulated image in the case of the $3 \mathrm{MeV}$ gamma beam. It is clearly possible to recognize the expected octagonal shape, due to the peculiar collimation system [8]. The image results enlarged along $\mathrm{x}$ axis due to the fact that the gamma beam impinges onto the scintillator target at angle of $45^{\circ}$. During the operation phase of the GPI, this stretching effect can be easily corrected in post-processing without of the risk of introducing artifacts.

\section{Conclusions}

The design approach for the beam imager for ELI-NP-GBS was presented. The adopted solution consists of a scintillator target intercepting the gamma beam and a system, composed of a CCD camera and a related lens, capable of acquiring the light emitted by the target. An analytical model has been developed to predict the GPI performance and this model was validated by carrying out a set of experimental tests on a simple GPI prototype. Subsequently, the expected images provided by the GPI in case of ELI-NP-GBS beam were evaluated by performing a set of Monte Carlo simulations using Geant4 and a custom made paraxial ray-tracing code. The expected signal will allow us to obtain an image of the spatial distribution of the gamma beam, both in commissioning and operation phase, in a small amount of time $(\sim 1 \mathrm{~s})$ for the entire energy range. The final system is currently being assembled in our laboratories. 


\section{References}

[1] ELI-Extreme Light Infrastructure, 2014. http://www.eli-laser.eu/.

[2] ELI-Nuclear Physics, 2014. http://www.eli-np.ro/.

[3] O. Adriani, et al., Technical Design Report EuroGammaS proposal for the ELI-NP Gamma beam system, 2014. Published as arXiv:1407.3669 [physics.acc-ph].

[4] C. Vaccarezza, et al., A European proposal for the Compton gamma-ray source of ELI-NP, in: International Particle Accelerator Conference - IPAC'12, 2012, pp. 10861088.

[5] EuroGammas Association, 2014. http://www.e-gammas.com/.

[6] D.L. Balabanski, R. Popescu, D. Stutman, A. Tanaka, O. Tesileanu, C.A. Ur, D. Ursescu, N.V. Zamfir, New light in nuclear physics: The extreme light infrastructure, Europhys. Lett. 117 (2) (2017) 28001.

[7] V. Petrillo, A. Bacci, R. Zinati, Ben Alì, I. Chaikovska, C. Curatolo, M. Ferrario, C. Maroli, C. Ronsivalle, A.R. Rossi, L. Serafini, et al., Photon flux and spectrum of gamma-rays Compton sources, Nucl. Instrum. Methods Phys. Res. A 693 (2012) 109-116.

[8] G. Paternò, P. Cardarelli, M. Marziani, E. Bagli, F. Evangelisti, M. Andreotti, M. Gambaccini, V. Petrillo, I. Drebot, A. Bacci, C. Vaccarezza, L. Palumbo, A. Variola, A collimation system for ELI-NP gamma beam system - design and simulation of performance, Nucl. Instrum. Methods Phys. Res. B 402 (2017) 349-353.

[9] P. Cardarelli, M. Gambaccini, M. Marziani, E. Bagli, V. Petrillo, A. Bacci, C. Curatolo, I. Drebot, C. Vaccarezza, Monte Carlo simulation of a collimation system for lowenergy beamline of ELINP Gamma Beam System, Nucl. Instrum. Methods Phys. Res. B 355 (2015) 237-240.

[10] M. Lenzi, O. Adriani, S. Albergo, M. Andreotti, D. Berto, R. Borgheresi, G. Cappello, P. Cardarelli, R. Ciaranfi, E. Consoli, G. Di Domenico, F. Evangelisti, M. Gambaccini, G. Graziani, M. Marziani, L. Palumbo, G. Passaleva, M.G. Pellegriti, A. Serban, O. Starodubtsev, M. Statera, A. Tricomi, A. Variola, M. Veltri, A new-concept gamma calorimeter at ELI-NP, J. Instrum. 12 (02) (2017) C02051.

[11] M.G. Pellegriti, S. Albergo, O. Adriani, M. Andreotti, D. Berto, R. Borgheresi, G. Cappello, P. Cardarelli, E. Consoli, G. Di Domenico, F. Evangelisti, M. Gambaccini, G. Graziani, M. Lenzi, M. Marziani, L. Palumbo, G. Passaleva, G. Paternò, A. Serban, S. Squerzanti, O. Starodubtsev, A. Tricomi, A. Variola, M. Veltri, B. Zerbo, The nuclear resonance scattering calibration technique for the EuroGammaS gamma characterisation system at ELI-NP-GBS, J. Instrum. 12 (03) (2017) C03058.
[12] S. Agostinelli, et al., Geant4 - a simulation toolkit, Nucl. Instrum. Methods Phys. Res. A 506 (3) (2003) 250-303.

[13] M. Nikl, Scintillation detectors for X-rays, Meas. Sci. Technol. 17 (4) (2006) R37.

[14] P. Lecoq, Development of new scintillators for medical applications, Nucl. Instrum. Methods Phys. Res. A 809 (2016) 130-139. Advances in detectors and applications for medicine.

[15] M. Nikl, A. Yoshikawa, K. Kamada, K. Nejezchleb, C.R. Stanek, J.A. Mares, K. Blazek, Development of luag-based scintillator crystals - a review, Prog. Cryst. Growth Charact. Mater. 59 (2) (2013) 47-72.

[16] S. Baccaro, K. Blazek, F. de Notaristefani, P. Maly, J.A. Mares, R. Pani, R. Pellegrini, A. Soluri, Scintillation properties of yap:ce, Nucl.Instrum. Methods Phys. Res. A 361 (1) (1995) 209-215.

[17] V. Kalinnikov, E. Velicheva, Investigation of LYSO and GSO crystals and simulation of the calorimeter for comet experiment, Phys. Part. Nuclei Lett. 11 (3) (2014) 259268.

[18] Epic-Crystal, Shanghai, China. http://www.epic-crystal.com/.

[19] Jianming Chen, Liyuan Zhang, Ren yuan Zhu, Large size LSO and LYSO crystal scintillators for future high-energy physics and nuclear physics experiments, Nucl. Instrum. Methods Phys. Res. A 572 (1) (2007) 218-224. Frontier Detectors for Frontier Physics.

[20] R. Mao, L. Zhang, R.Y. Zhu, Optical and scintillation properties of inorganic scintillators in high energy physics, IEEE Trans. Nucl. Sci. 55 (4) (2008) 2425-2431.

[21] Ren-Yuan Zhu, The next generation of crystal detectors, Proc. SPIE 9593 (12) (2015) 9593-9593.

[22] H. Liu, A. Karellas, L.J. Harris, C.J. D'Orsi, Methods to calculate the lens efficiency in optically coupled CCD X-ray imaging systems, Med. Phys. 21 (7) (1994) 1193-1195.

[23] Varian, Palo Alto, California, USA. http://www.varian.com/.

[24] Metaltronica, Pomezia, Rome, Italy. http://www.metaltronica.com/it/.

[25] Nikon, Minato, Tokyo, Japan. http://www.nikon.com/.

[26] Diffraction Limited, Ottawa, Canada. http://diffractionlimited.com/.

[27] G.G. Poludniowski, G. Landry, F. DeBlois, P.M. Evans, F. Verhaegen, SpekCalc: A program to calculate photon spectra from tungsten anode X-ray tubes, Phys. Med. Biol. 54 (2009) N433.

[28] MATLAB, version 8.6 (R2015b), The MathWorks Inc., Natick, Massachusetts, 2015. 